\title{
Study on Damping Characteristics of Automobile Shock Absorber based on Fluid-Structure Interaction Simulation Xin Wen $\mathrm{Bi}^{1}$, Shao Cheng Song ${ }^{1, a}$
}

${ }^{1}$ College of Information Technology and Media, Beihua University, 132013, Jilin City, China

\begin{abstract}
The automobile's better performance of suspension system can improve good performance of power and mobility so that it has good driving operation, low vibration and low noise, comfortable driving environment. In this paper, the test to verify the simulation results of the damping characteristics by comparing the simulation external characteristics with the experimental external characteristic and analyzing the advantages and disadvantages of the simulation method.
\end{abstract}

Keywords: Shock absorber, Damping characteristics, Simulation.

\section{Introduction}

In the modern automobile industry, in addition to automotive safety, economy and power requirements in terms of comfort, reliability, durability and safety requirements are also increasing. Superior driving maneuverability, ride a good environment, low vibration and noise, it has become an important symbol of modern high-quality cars. The more superior performance suspension means the vehicle can make its full power and flexibility, its performance improved, therefore the suspension device becomes critical.

It ensures an elastic contact between the wheel and the car bearing system, and the ability to transfer loads, vibration damping, to ease the impact on the body and adjusting the height position of the moving body. Through the damper and damping effect of damping material on the suspension, the relative between the wheel and the vehicle body vibration damping off, therefore, the damper performance directly determines the performance of the suspension device. In recent years, electronically controlled variable damping shock absorber in foreign countries has been widely used. However, the current domestic and foreign design or shock absorber to complete a single design and the design of the task, with the rapid development of the automotive industry, it is necessary shock absorber designed to respond quickly to market needs, so that in a short period of completion time and cost savings. Shock Absorber performance quality of the car is critical, it directly affects the performance of dynamic resistance nits of vehicle dynamics.

The traditional design approach is usually damper according to the precision requirements and requirements of the vehicle, based on the accumulated experience to select the design parameters of the shock absorber, manufactured test sample, through experiments indeed whether the requirements of the design of the damper. This approach often been repeated to adjust the design parameters, the repeated manufacture of experimental samples, repeated experiments and adjustments, so that the design cost becomes expensive.

With the FEM (Finite Element Method, FEM) and computational fluid dynamics (Computational Fluid Dynamics, CFD) continues to develop, so reducing the shock absorber design and development of experimental dependence, reducing damper parameters different samples the cost of manufacturing and real vehicle test and shorten the development cycle, improve design efficiency damper, it becomes very meaningful.

\section{Research status of automobile shock absorber}

Domestic research with respect to foreign automobile shock absorber late start, but the beginning of the design methods are traditional rely on experiments to determine the design parameters. With the continuous development of computer technology as well as to foreign Shock Absorber continuous learning, Chinese scholars have gradually begun to use CAD/CAE software for shock absorber design and development. The basic process of this method is: the establishment of the actual structure of the damper according to its geometry and geometric models into analysis software simulation analysis to obtain the damping characteristics of the shock absorber, the damping characteristics according to the simulation analysis obtained it steering stability, ride comfort and ride comfort and other performance to improve it and optimize. The method of finite element software simulation of the shock absorber, shock absorber design and development of great significance. With the rapid development of China's auto industry, Chinese scholars study the deepening of shock absorber technology, to explore research can improve damper performance technology. To improve the performance of the shock absorber, it is necessary to make a study of the previous

\footnotetext{
a Corresponding author: Shaochengsong007@126.com
} 
damper system analysis and summary for future research on this aspect of the work of scholars who have some enlightening. Mechanics (Computational Fluid Dynamics, CFD) and finite element analysis (Finite Element Analysis, FEA) and other methods to build and solve the hybrid model has become possible. Shock Absorber is a complex nonlinear systems dynamics model for the establishment of the shock absorber has been a concern of scholars. Foreign scholars on the shock absorber, mainly through its three modeling methods research: the physical parameters of the model, the model equivalent parameters and non-parametric models.

\section{The basic theory of fluid - solid coupling}

Through the analysis of the shock absorber above the work process, we can see this working process belongs to the category of fluid-structure interaction. Coupling, refers to the coupling between fluid and solid between each other, resulting in a series of phenomena. People in order to investigate the influence of the flow field, the deformation behavior of a variety of solids and solid-bit form convection properties produced interactive relationship between the two, thereby generating a new branch of mechanics - Fluid - solid coupling dynamics.

Coupling Dynamics is a solid stream involves both CFD (Computational Fluid Dynamics, CFD) and relates to solid mechanics (Computational Solid Mechanics, CSM) is a discipline, shown in Figure 2.2. Fluid-structure coupling Problem solving involves both solid, but also relates to fluid solver. Because taking their own characteristics, and solving these two aspects, it makes fluid-structure coupling problem solving results closer to the physical laws of the phenomenon itself. Since the fluid-structure coupling phenomenon exists widely in various fields, and therefore, fluid-structure interaction study has important significance. In recent years, with the continuous development of science and technology, using software simulation technique for solving fluid-structure interaction and design is becoming increasingly widespread in engineering.

\section{CFD numerical simulation method}

CFD (Computational Fluid Dynamics) is referred to as computational fluid dynamics. Computational fluid dynamics yes a branch of mechanics, numerical methods it and data visualization technology combine the relevant physical phenomena flow, heat transfer and other simulation analysis. The basic idea of CFD are: the original continuous physical field in time and spatial domain, a series of discrete points with variable set of values instead, and set up to reflect these variables of discrete points by playing certain principles and modalities relations between algebraic equations and then solving algebraic equations to obtain an approximation of field variables.
CFD technology is in conformity with the fluid control equations, numerical simulation of fluid flow. Fluid equations have the energy conservation equation, mass conservation equation, momentum conservation equation. CFD numerical simulation of flow distribution can be obtained for each parameter field and their circumstances change over time. CFD technology can compensate for the lack of theoretical analysis and experimental measurement methods.

Based on the working process of the shock absorber, coupled with a solid approach flow simulation analysis, that both the fluid analysis result to the analysis of solid structures, but also the results of the analysis of solid structures (such as displacement, velocity and acceleration) response to the fluid transfer analysis. In ANSYS WorkBench platform with ANSYS and ANSYS CFX FSI simulation joint general technical route. Among them, the establishment of three-dimensional solid model of this paper chose CATIA software, then geometry processing in ANSYS WorkBench geometry; meshing selection grid before choosing HyperMesh software, because of the simple structure of the solid portion, larger mesh can achieve accuracy, the fluid part of the complex structure, requiring a finish painting hexahedral grid to meet the accuracy requirements; set herein solid simulation model selection ANSYS WorkBench of Transient structural (ANSYS) module, the fluid domain calculations herein choice fluid Flow (CFX).

\section{Simplified simulation system}

This binocular damper hydraulic system consisting of a closed system, the internal fluid motion by the pull rod reciprocating motion caused. So the model should include the complete recovery of the damper valve, circulation valve, compensating valves and compression valve and mesh constraint set, driven by movement of the piston rod assembly valve stationary no-slip wall cavity, gas chamber (reservoir chamber above) and the gas chamber and the reservoir chamber of the gas-liquid interface, and so on. However, the establishment of a complete damper model brings two problems: First, they must use the grid to simulate the dynamic motion of the piston driven movement around the oil, which causes the system to simulate the mesh quality requirements will be higher setting parameters is complex, and most parameters are set empirically, there is no accurate theoretical basis, after setting the simulation is also very easy to make mistakes; second is to greatly increase the number of grid computing will consume a lot of time and resources, the performance requirements of the computer will be high. As can be seen from the shock absorber structure, the number of circulating valve and compensating valve little throttling effect at work with respect to the recovery valve and the compression valve is negligible. Therefore, only the recovery valve and compression were established flow field mesh valve. 


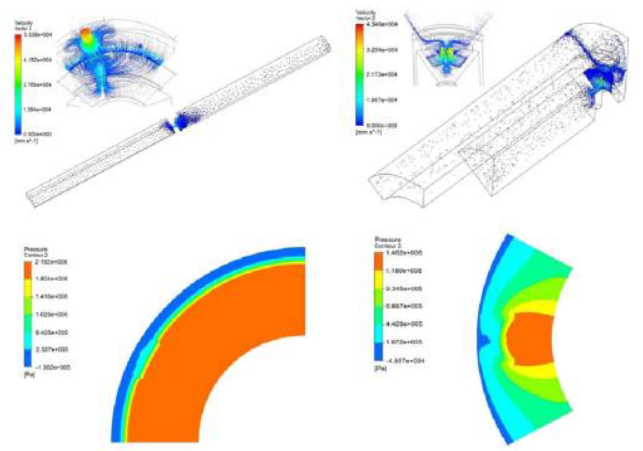

Fig. 1 Speed damping valve vector Fig. 2 Fluid-structure coupling surface pressure contours

\section{Summary}

Depending on performance shock absorbers, obtained by experiment specific performance parameters and conditions of this test piece, and the experiments of the speed curve and shown in FIG workers and simulation results were compared, the results can be seen by comparing the experimental results agree well with the simulation results to verify the feasibility of this simulation method, and to simulate errors and experimental results appear, error analysis.

\section{Acknowledgement}

1. Ministry of Education Chunhui projects: vehicle vibration damping characteristics, project number 104900150.

2. National Social Science Fund Project: integration and application of multi-modal intelligence crisis in the city emergency management (item number: 15BTQ062)

\section{References}

[1] Chen Jiarui, Jilin University, Department of Automotive Engineering car construction. China Communications Press, 2002.

[2] DIXON J. The shock absorber handbook [M]. John Wiley \& Sons, 2007.

[3] Li Shimin, Lv Zhenhua car telescopic hydraulic dampers technology development of automotive technology, 2001, 8): 10-6.

[4] Lv Zhenhua, Wu Zhuoan, Li Shimin finite element simulation analysis of nonlinear characteristics of the throttle damper mechanical strength, 2004, 25 (6): 614-20.

[5] Studies valve throttle characteristic Li Xiaobo, Shen Sheng season, ROCKETS, et al restoration based on the shock absorber fluid-solid coupling analysis of mechanical manufacturing and automation, 2010, 40 (4): 20-2.

[6] He Ping Simulation Technology of Automotive Shock Absorber Dynamic characteristics; Beijing: Beijing Institute of Technology, 2010.

[7] Feng Xuemei, Liu Zuo people inside the hydraulic shock absorber characteristic finite element analysis of the behavior of the distribution of Wuhan University of Technology: Information \& Management Engineering, 2002, 24 (5): 121-4.

[8] He Ping, Gu Liang, Xin Guoguo, et al annular damper valve large deflection precision analytical expression Beijing Institute of Technology, 2009, 6): 510-4.

[9] Lv Zhenhua, Li Shimin, head Liu Zhen, et al Experimental Research telescopic hydraulic dampers operating characteristics of Automotive Engineering, 2005, 27 (2): 203-8.

[10] Chen Yijie, Gu Liang, Yang Zhanhua, et al deformation of throttle slice large deflection damper mechanical design and manufacturing, 2008, 10): 90-2.

[11] $\mathrm{Xu}$ Zhongming, Zhang Yufeng, Yu Feng, et al variable damping shock absorber outer Simulation and Test of Chongqing University: Natural Science Edition, 2010, 33 (9): 29-34.

[12] The throttle plate and throttle opening week of the Great Wall research, Gu superimposed light absorber fluid machine, 2006, 34 (7): 19-23.

[13] Zhao Feng automobile shock absorber damping characteristics based on MATLAB environment simulation analysis; Huazhong University of Science and Technology, 2007. 